\title{
EDITORIAL
}

\section{Integrating Traditional Medicine with Biomedicine Towards a Patient-Centered Healthcare System}

\author{
XU Hao (徐 浩) and CHEN Ke-ji (陈可冀) ${ }^{2}$
}

In this new age of scientific developments, biomedicine makes it possible for us to draw precise pictures of the internal workings of the human body, measure tiny metabolic reactions, exchange organs from one person to another, and even grow babies in test tubes. These accomplishments have enabled us to alter successfully and dramatically the natural history of many diseases. Nobody can detract from these achievements, and it is a matter of course for biomedicine to be regarded as conventional medicine or mainstream medicine. However, biomedicine is at its limits nowadays when confronting degenerative diseases, stress-related diseases, and most chronic diseases, which are more related to the way we think and live than to bacteria and viruses. Most notably, biomedicine lacks reference to the self-healing capacity of the human mind and body and focuses on parts rather than the whole, treatment rather than prevention, the suffering disease rather than the diseased person.

Confronted with these problems, more and more far-sighted western scholars began to lay their eyes on traditional medicine (TM). According to the definition from the World Health Organization (WHO), TM refers to the knowledge, skills, and practices based on the theories, beliefs, and experiences indigenous to different cultures, used in the maintenance of health and in the prevention, diagnosis, improvement, or treatment of physical and mental illnesses. TM therapies include medication therapies - if they involve use of herbal medicines, animal parts and/or minerals - and nonmedication therapies - if they are carried out primarily without the use of medication as in the case of acupuncture, manual therapies and spiritual therapies. In countries where the dominant healthcare system is based on biomedicine, or where TM has not been integrated into the national healthcare system, TM is often termed "complementary", "alternative", or "non-conventional" medicine or used interchangeably with $\mathrm{TM}^{(1,2)}$.

It is well established that TM plays an important role in healthcare for the majority of the population living in developing countries. In fact, TM was even the only healthcare system available to the prevention and treatment of diseases in different cultures for centuries. For example, Chinese medicine (CM) has a history of thousands of years and has made great contributions to the health and well-being of the people and to the maintenance and growth of the population. Currently, more than $90 \%$ of the urban and rural Chinese population has sought for CM in their lifetimes ${ }^{(3,4)}$. In Africa, up to $80 \%$ of the population uses TM to help meet their healthcare needs. In Asia and Latin America, populations continue to use TM as a result of historical circumstances and cultural beliefs. Meanwhile, in many developed countries, complementary and alternative medicine (CAM) is becoming more and more popular. The percentage of the population that has used CAM at least once is $48 \%$ in Australia, $70 \%$ in Canada, $42 \%$ in USA, $38 \%$ in Belgium, and $75 \%$ in France ${ }^{(2)}$. In addition, information derived from various systems of TM even plays an important role in drug discovery ${ }^{(5)}$, and over $50 \%$ of the best-selling pharmaceuticals we are using today are derived from natural products.

TM covers a wide variety of therapies and practices, such as CM, Japan Kampo medicine, Indian Ayurveda medicine, Unani medicine, and various forms of indigenous medicine. In this issue, Dr. Motoo, et $\mathrm{al}^{(6)}$ introduce the history and current status of Japan Kampo medicine, from which the experience of integrating Kampo into modern medicine in Japan is quite worthwhile to learn. Dr. Patwardhan, et $\mathrm{al}^{(7)}$ review the basics of Rasayana therapy from Ayurveda and the published researches on different Rasayana drugs for specific health conditions, which may provide candidates for the development of antioxidants and immunomodulators. Dr. Yesilada ${ }^{(8)}$ discusses the contribution of Unani medicine or Islamic medicine in the healthcare system of the Middle East. The rich tradition of the Middle East communities in the utilization of herbal

CThe Chinese Journal of Integrated Traditional and Western Medicine Press and Springer-Verlag Berlin Heidelberg 2011

1. National Integrative Medicine Center for Cardiovascular Disease, China-Japan Friendship Hospital, Beijing (100029),

China; 2. Xiyuan Hospital, China Academy of Chinese Medical Sciences, Beijing (100091), China

Correspondence to: Prof. CHEN Ke-ji, Tel: 86-10-62860894,

E-mail: chen.keji@gmail.com

DOI: 10.1007/s11655-011-0641-2 
remedies as well as diverse spiritual techniques for treating various disorders is very impressive. Dr. Omonzejele, et $\mathrm{al}^{(9)}$ show us metaphysical and value underpinnings of TM in West Africa. His investigation indicated that a large number of Africans consider sicknesses as having both physical and supernatural components. Although the strategies used to checkmate spiritual causes, such as the use of sacrifices and the transference of illnesses to animals and other objects, seem to be a little mysterious and unimaginable, its positive aspects might still be taken as references by modern psychotherapy and mind-body medicine.

It is indisputable that the ultimate goal of any medicine is to improve health of mankind and enhance the therapeutic effect in preventing and treating diseases. In this sense, any medicine should be patient-centered. All kinds of available therapeutic approaches, as long as safe and effective, should be embraced in the same healthcare family. Under this circumstance, the concept and practice of integrative medicine (IM) emerges as the times require ${ }^{(10-12)}$. TMs are most precious treasures from our ancestors to build a patient-centered healthcare system. WHO has been promoting TM as a source of less expensive, comprehensive medical care, especially in developing countries ${ }^{(2)}$. However, most TMs have not been successfully integrated into their national healthcare systems. For example, in Africa and Latin America, TMs are completely separated from conventional medicine. There is no communication, no integration.

Worldwide, only China, the Democratic People's Republic of Korea, the Republic of Korea and Viet Nam can be considered to have attained an integrative system ${ }^{(2)}$. Especially in China, the integration of $\mathrm{CM}$ and Western medicine has been explored for more than a century. The experience has exemplified the tolerance, dependence, and assimilation of not only two medicines but also other science disciplines in $\mathrm{IM}^{(3)}$, and provided a paradigm for worldwide $\mathrm{IM}^{(13,14)}$. Most importantly, treatment modalities that are not considered part of mainstream medicine equally deserve evaluation and research, and should be considered as opportunities that serve to enrich and perfect conventional medicine rather than be viewed as competitors or disregarded. Some characteristics of CM, such as the concepts of seeing the body as a whole, preventive treatment of disease (Zhi Wei Bing, 治未病), living in harmony with the environment (Tian Ren Xiang Ying, 天人 相应), treatment based on CM pattern differentiation, and compound prescriptions with synergistic effect, will also provide biomedicine with new perspectives.

Of course, there are still many difficulties in integrating TM into the overall healthcare systems partly due to the insufficient evidence in clinical settings and the problems in safety and quality control. Nevertheless, it is our dream that, in the future, diverse modalities, such as herbal medicine, acupuncture, moxibustion, Tuina bodywork, spiritual therapies, chiropractic, homeopathy, osteopathy, naturopathy, and many other TM therapies, as well as biomedicine can work in conjunction with each other as part of a unified team rather than in competition. This integrative approach will ultimately lead to a safer, more convenient and effective patient-centered healthcare system.

\section{REFERENCES}

1. World Health Organization. General guidelines for methodologies on research and evaluation of traditional medicine. 2000. http://whqliboc. who.int/hq/2000/ WHO_EDM_TRM_2000.1.pdf.

2. World Health Organization. Traditional medicine strategy 2002-2005. 2002. http://whqlibdoc.who.int/hq/2002/ WHO_EDM_TRM_2002.1.pdf.

3. $\mathrm{Xu} \mathrm{H}$, Chen KJ. Integrative medicine: the experience from China. J Altern Complem Med 2008;14:3-7.

4. Lu AP, Ding XR, Chen KJ. Current situation and progress in integrative medicine in China. Chin J Integr Med 2008;14: 234-240.

5. Fabricant DS, Farnsworth NR. The value of plants used in traditional medicine for drug discovery. Environ Health Perspect 2001;109 (Suppl 1):69-75.

6. Motoo Y, Seki T, Tsutani K. Traditional Japanese medicine, Kampo: its history and current status. Chin J Integr Med 2011;17:85-87.

7. Balasubramani SP, Venkatasubramanian P, Kumar SK, Patwardhan B. Plant-based Rasayana drugs from Ayurveda. Chin J Integr Med 2011;17:88-94.

8. Yesilada E. Contribution of traditional medicine in the health care system of the Middle East. Chin J Integr Med 2011;17:95-98.

9. Omonzejele PF, Maduka C. Metaphysical and value underpinnings of traditional medicine in West Africa. Chin $\mathrm{J}$ Integr Med 2011;17:99-104.

10. Rees L, Weil A. Integrated medicine. BMJ 2001;322:119-120.

11. Eisenberg D. Reflections on the past and future of integrative medicine from a lifelong student of the integration of Chinese and Western medicine. Chin J Integr Med 2011;17:3-5.

12. Weil A. The state of the integrative medicine in the U.S. and western world. Chin J Integr Med 2011;17:6-10.

13. Dobos G, Tao I. The model of Western integrative medicine: the role of Chinese medicine. Chin J Integr Med 2011;17:11-20.

14. Robinson N. Integrative medicine-traditional Chinese medicine, a model? Chin J Integr Med 2011;17:21-25.

(Received December 6, 2010) Edited by WANG Wei-xia 